\title{
Two-sided ultrasonic surface rolling process of aeroengine blades based on on-machine noncontact measurement
}

(C) The Author(s) 2020. This article is published with open access at link.springer.com and journal.hep.com.cn

\begin{abstract}
As crucial parts of an aeroengine, blades are vulnerable to damage from long-term operation in harsh environments. The ultrasonic surface rolling process (USRP) is a novel surface treatment technique that can highly improve the mechanical behavior of blades. During secondary machining, the nominal blade model cannot be used for secondary machining path generation due to the deviation between the actual and nominal blades. The clamping error of the blade also affects the precision of secondary machining. This study presents a two-sided USRP (TS-USRP) machining for aeroengine blades on the basis of on-machine noncontact measurement. First, a TS-USRP machining system for blade is developed. Second, a 3D scanning system is used to obtain the point cloud of the blade, and a series of point cloud processing steps is performed. A local point cloud automatic extraction algorithm is introduced to extract the point cloud of the strengthened region of the blade. Then, the tool path is designed on the basis of the extracted point cloud. Finally, an experiment is conducted on an actual blade, with results showing that the proposed method is effective and efficient.
\end{abstract}

Keywords aeroengine blades, on-machine noncontact measurement, point cloud processing, path planning, surface strengthening

Received September 17, 2019; accepted October 28, 2019

Shulei YAO, Xian CAO, Shuang LIU (囚), Kaiming ZHANG, Xiancheng ZHANG (ه)

Key Laboratory of Pressure Systems and Safety (Ministry of Education), East China University of Science and Technology, Shanghai 200237, China

E-mails: shuangliu@ecust.edu.cn; xczhang@ecust.edu.cn

Congyang GONG, Chengcheng ZHANG

AECC Commercial Aircraft Engine Co., Ltd., Shanghai Engineering Research Center for Commercial Aircraft Engine, Shanghai 201108, China

\section{Introduction}

As crucial parts of an aeroengine, blades play a vital role in energy conversion and flight power supply. Blades are prone to various defects, such as cracks, distortion, wear, and impact dents, due to long-term operation at high temperature, complex stresses, and extreme environment conditions [1,2]. Statistics show that blade damage and failure account for approximately $70 \%$ of component failures in aeroengines. Hence, blades have limited life. Prolonging the service life of aeroengine blades is important for the aviation industry.

Strengthening the blade surface can improve the residual compressive stress, inhibit the formation and propagation of cracks, and improve the fatigue life of the blade $[3,4]$. Generally, surface treatment techniques applied to aerospace components mainly include shot peening (SP), laser shock processing (LSP), and rolling process (RP) $[5,6]$. Surface treatment, which is applied to the actual blade, mainly includes SP and LSP $[7,8]$. SP has been developed for approximately 100 years. As a cold working process, SP is applied to reduce the stress concentration of metal parts (such as blade disc interface) and improve their surface performance [9]. Although SP is relatively inexpensive and widely applied, uniform SP intensity across components is not guaranteed, residual compressive stresses are limited in depth, and SP results in a roughened surface [10]. Consequently, LSP has been rapidly developed as an alternative novel surface treatment technique. LSP was successfully applied to the fan/compressor blades of an aeroengine in 1997. LSP can produce sufficient residual compressive stress layers and remarkably improve the fatigue performance of the workpiece [11]. Furthermore, LSP has a simple operation, local treatment, and short processing duration [12]. However, it is a relatively expensive surface treatment method and has a complicated working environment [13]. Proposed by Wang et al. [14], ultrasonic surface rolling process (USRP) is a novel nanostructured surface treatment technique that combines 
static extrusion and dynamic impact to generate intensive plastic deformation and strengthen material surfaces. Studies [15-17] have shown that USRP can improve the yield strength of material surface layers, introduce large residual compressive stress, and reduce surface roughness. Thus, USRP is an effective surface strengthening method. Relevant research on USRP has focused on metallic test piece strengthening, which has not been applied to aeroengine blades.

Aeroengine blades are typical thin-walled structure parts with poor stiffness and easy deformation. Static extrusion and dynamic impact are imposed on the blade when it is processed through USRP. Thus, applying USRP to a single surface of the blade causes deformation. Two-sided machining is used to prevent the deformation of thinwalled parts, and it mainly involves milling, grinding, and polishing. Song et al. [18] proposed a path planning method for turbine blades with twin-tool milling and conducted an experiment on blade twin-tool machining using a nine-axis machine tool. Results showed that machining efficiency was immensely improved. Li et al. [19] and Zhang et al. [20] presented a machine for the synchronous grinding and polishing of the two sides of the blade to improve efficiency and machining accuracy. Twosided machining methods, such as SP and LSP, were used for surface strengthening. Kopp and Schulz [21] developed a new double-sided simultaneous SP and proved its feasibility through experiments. Sakhvadze et al. [22] described the principles of two-sided LSP and established its finite element model. However, no study has been conducted on two-sided USRP (TS-USRP) machining of aeroengine blades.

Manufacturing errors are inevitable during blade forming because blades are thin-walled parts with uncertain free-form surfaces. Repeated clamping of blades during secondary machining results in deviation between the actual and theoretical positions. Therefore, a nominal computer-aided design model of blades cannot be directly used for secondary machining path generation [23]. With the rapid development of reverse engineering technology, on-machine measurement (OMM) has been widely used in secondary numerical control (NC) machining to achieve accuracy. At present, 3D measurement of workpieces mainly includes contact and noncontact measurement methods [24]. The contact measurement method uses a probe to measure the workpiece surface and has mature technology, high precision, and strong reliability; however, it has low efficiency and may cause dead angles during the measurement of uncertain free-form surfaces. The noncontact measurement method prevents the probe from touching the surface during workpiece measurement and has high real-time performance and fast measurement speed. OMM has been applied to the processing and testing of free-form surfaces. Xiao et al. [25] successfully applied on-machine contact measurement (OMCM) to the belt grinding of a propeller blade and proved its effectiveness and practicability. Huang et al. [26] presented an integrated machining deviation compensation strategy combined with OMCM to obtain the coordinates of sampling points and reconstruct the machined surface. Nishikawa et al. [27] developed a noncontact-type OMM system for measuring the cross sections of a turbine blade and verified its effectiveness. The contact measurement method has low-efficiency technology, although it is commonly used for the measurement and inspection of workpieces. The noncontact measurement method is gradually being applied for the measurement of free-form workpieces.

This study presents a TS-USRP for aeroengine blades based on on-machine noncontact measurement (OMNCM). In this method, the principle of USRP is applied to strengthen the aeroengine blade, and a set of TS-USRP machining system for the blade is developed. OMNCM is used to obtain the point cloud of the blade, and a series of point cloud processing steps is performed. Subsequently, the local region point cloud of the blade is extracted using the proposed local point cloud automatic extraction algorithm, and the $\mathrm{NC}$ tool path is designed on the basis of the extracted point cloud. Then, an experiment is conducted on an actual blade. The main contributions of this study are as follows:

1) A TS-USRP machining system for aeroengine blades is developed. Blades are easily deformed during strengthening because they are typical thin-walled workpieces. Two-sided simultaneous processing of the blade, which can offset the force exerted on a single surface of the blade and avoid its deformation, is adopted. Two-sided machining can improve the efficiency of blade strengthening.

2) OMNCM is used to ensure the accuracy of the TS-USRP machining tool path. The blade clamped on the machine tool is directly measured using a structured lightbased 3D scanning system. Few common marked points are observed at the junction of the suction and pressure sides due to the thin-walled structure of the blade. Thus, point cloud registration of the entire blade is difficult [28]. The marked points of the blade are extended to scan the chuck, fixture and blade, and the common marked points of the chuck and fixture are used to realize the global registration of the blade point cloud. In addition, a local point cloud automatic extraction algorithm is presented to extract the local region point cloud of the blade.

3) An experiment on an actual aeroengine blade is conducted. This study applies TS-USRP to the actual blade and verifies the feasibility and efficiency of the proposed method.

The remainder of this paper is organized as follows: Section 2 presents the framework of the proposed method; Section 3 introduces the TS-USRP machine tool system; Section 4 uses OMNCM to obtain the point cloud of the blade and conducts point cloud processing; Section 5 
designs the TS-USRP machine tool path of the blade. Section 6 conducts an experiment on the actual blade; and Section 7 provides the conclusion and future work.

\section{Framework}

Aeroengine blades are thin-walled parts with a complex structure. The structure of the blade includes the suction and pressure sides, the leading (LE) and trailing (TE) edges and the tenon, as shown in Fig. 1. The suction/pressure sides are a free-form surface connected by the LE and TE. The tenon is located at the blade root, which is an important part that connects the blade to the blade disc and bears immense force when working.

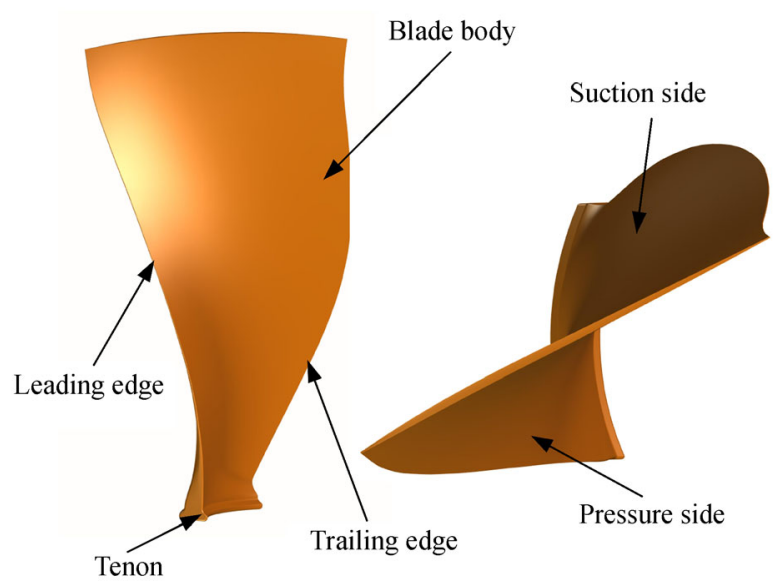

Fig. 1 Structure of the blade.

According to the early research, the manufacturing error of the blade root is the primary cause of the deviation between the actual blade and the nominal blade, and the blade profile is accurate. Figure 2(a) shows the manufacturing error of the blade after the blade root of the actual blade is aligned with that of the nominal blade. The clamping error of the blade is mainly reflected in the position error and the angular error in the $x / y / z$ direction after the blade is clamped, as shown in Fig. 2(b).

TS-USRP machining of the blade based on OMNCM is shown in Fig. 3. First, the local region of the blade to be strengthened should be identified. The nominal blade cannot generate the $\mathrm{NC}$ tool path due to the deviation between the actual and nominal blades and the manufacturing errors during blade forming. The repeated clamping of blades leads to non-coincidence between the actual machining and theoretical positions during secondary machining. Thus, OMNCM is used to obtain the point cloud of the blade. Then, point cloud preprocessing is required because of the integration of noise and redundant data in the raw point cloud, and the local region of the point cloud to be strengthened is extracted. Subsequently, fitting

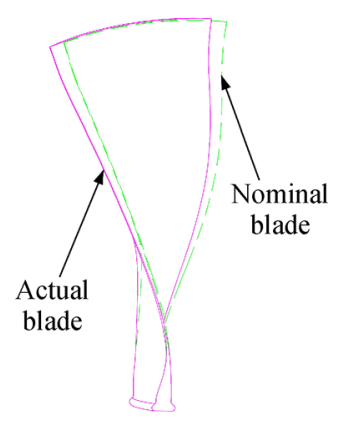

(a)

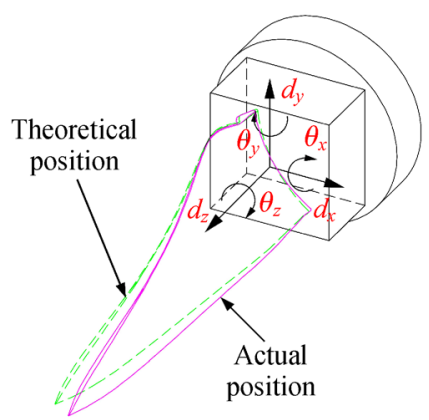

(b)
Fig. 2 Source of machining errors. (a) Manufacturing error of the blade; (b) clamping error of the blade.

and interpolation are required to generate an accurate and smooth path because the obtained point cloud is scattered. Finally, the tool path is generated and applied to the TS-USRP machining system of the blade.

\section{TS-USRP machine tool system}

\subsection{Principle of USRP}

USRP is a surface treatment technique that combines ultrasonic impact with rolling. The principle of USRP is shown in Fig. 4, and its working process is expressed as follows: First, an ultrasonic generator provides ultrasonic current at a certain frequency to the transducer; second, the ultrasonic transducer converts electrical oscillation into mechanical vibration with the same frequency, and the ultrasonic horn amplifies the mechanical vibration of the transducer and sends it to the rolling head; finally, the rolling head transmits static extrusion and ultrasonic shock vibration to the moving workpiece surface that is to be machined. Large elastoplastic deformation of the processed material occurs under the impact and extrusion of the rolling head, and the plastic flow of the metal flattens the bumps of the workpiece surface and fills the pits. Thus, the roughness of the machined surface is reduced considerably, and residual compressive stress is introduced $[29,30]$.

\subsection{TS-USRP machine tool system of the blade}

Considering that the blade is a thin-walled part with a freeform surface, the rolling head exerts a certain static extrusion and dynamic impact on the blade surface during USRP machining. Hence, deformation occurs when only one side of the blade is machined through USRP, especially when it is machined near the blade edge and tip. Thus, USRP machining on one side of the blade seriously affects machining accuracy. Two-sided synchronous USRP machining for blade strengthening is 


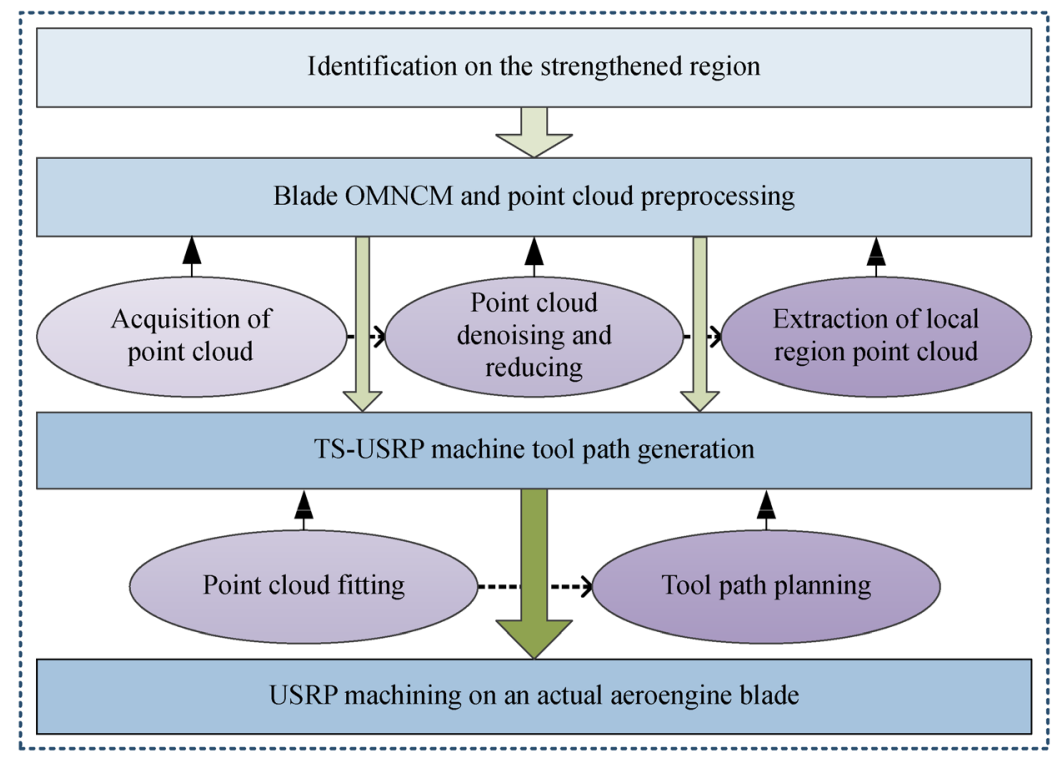

Fig. 3 TS-USRP machining of the blade based on OMNCM.

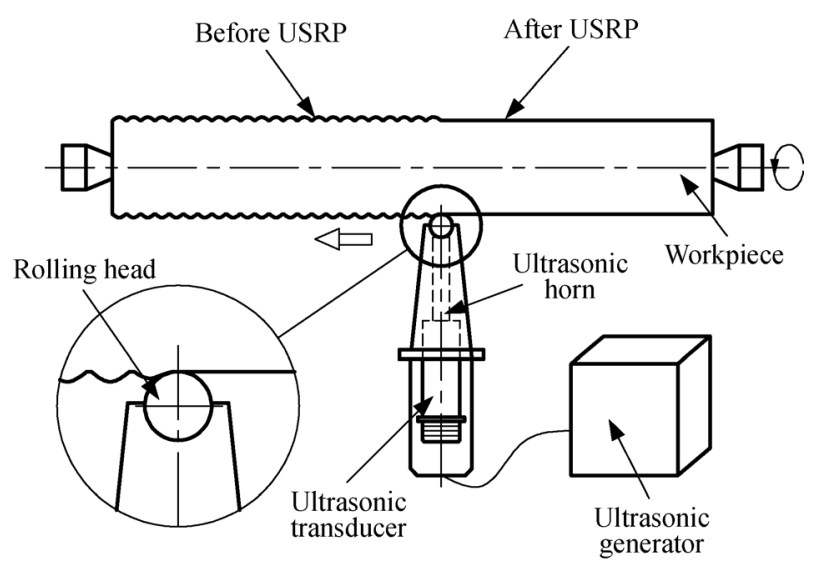

Fig. 4 Principle of USRP.

proposed, and a set of TS-USRP machine tool system is designed to solve this problem (Fig. 5). The system distribution is bilaterally symmetrical and consists of a machine bed, a spindle, a spindle movement system, a handle, a blade special fixture system, TS-USRP tools, and a USRP tool movement system. The blade can move in a straight line along the $z$-axis and rotate around the $z$-axis, and the TS-USRP tools can move along the $x$ - and $y$-axes. During machining, the ultrasonic generators on both sides were adjusted to the same frequency and amplitude. In addition, the handle holds the tip of the blade, and TS-USRP tools simultaneously act on the blade surface, thus offsetting the static extrusion and dynamic impact produced by a single USRP tool. In this manner, deformation is avoided during TS-USRP machining, and the efficiency of blade strengthening is improved significantly.

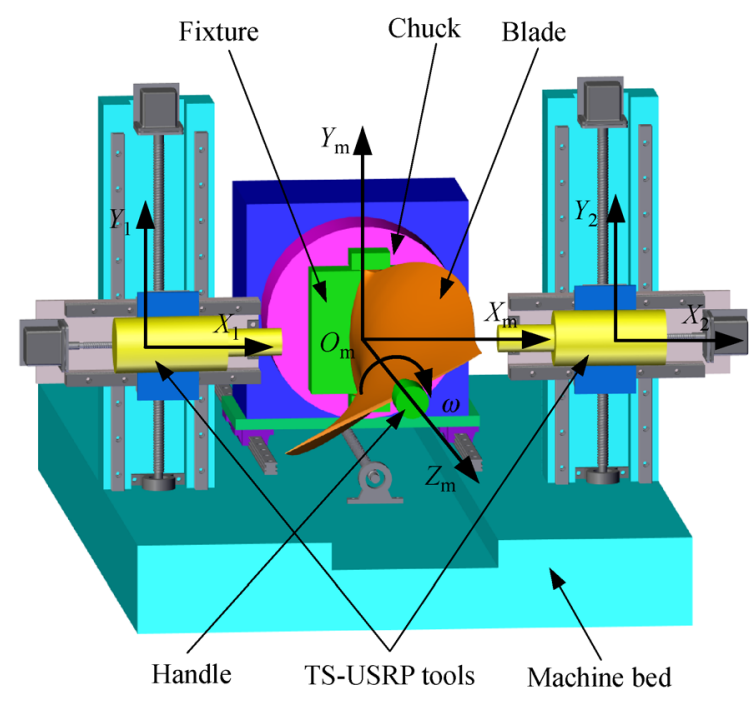

Fig. 5 Blade TS-USRP machine tool system.

\section{Blade OMNCM and point cloud processing}

\subsection{Acquisition of point cloud}

This study adopts OMNCM to obtain the point cloud of the blade by using a structured light-based 3D scanning system. The principle of OMNCM is shown in Fig. 6. The blade is scanned by the 3D scanning system, and the obtained point clouds are registered using common marked points. Considering that the blade is a thin-walled part, the suction and pressure sides are connected by the LE and TE, as shown in Fig. 1. As a result, few common marked points are observed between the suction and pressure sides, which 


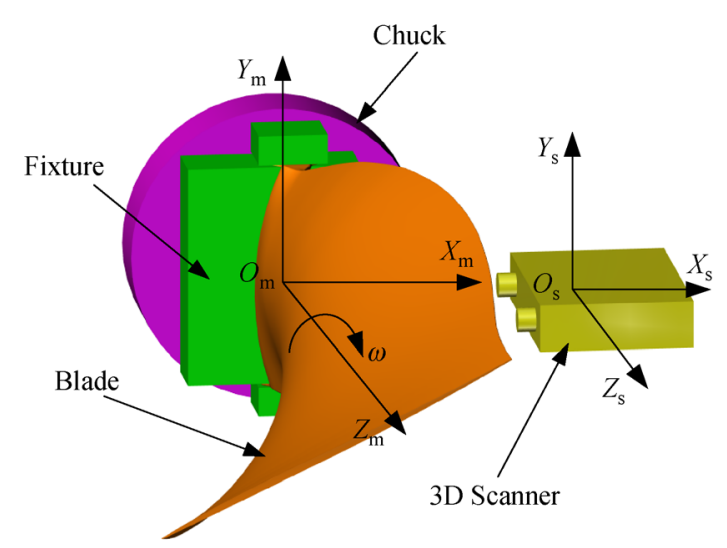

Fig. 6 Principle of OMNCM.

can be used during blade point cloud registration, as shown in Figs. 7(a) and 7(b). Thus, scanning a single blade cannot accurately register the point cloud of the entire blade, as shown in Fig. 7(c). On this basis, this study extends the blade marked points. This process scans the blade, fixture, and chuck as a whole, and the blade point cloud is registered using the marked points on the chuck and fixture on the basis of the invariance of their spatial characteristics, as shown in Fig. 8. The point cloud of the chuck and fixture is removed to obtain the blade point cloud.

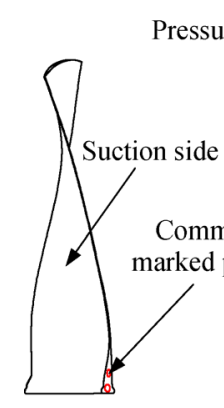

(a)

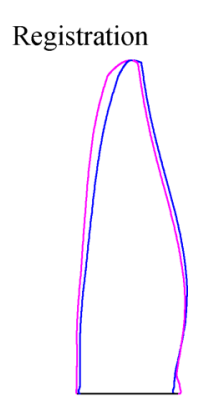

(c)
Fig. 7 Scanning a single blade. (a) One side of the blade; (b) another side of the blade; (c) registration of the point cloud of the entire blade.

\subsection{Point cloud preprocessing}

The coordinates of the raw point cloud measured by the 3D scanning system are based on the coordinate system of the 3D scanner. Hence, the coordinate system of the raw point cloud should be converted into the machining coordinate system. Define a point in the raw point cloud as $P_{i}=\left(x_{i}, y_{i}, z_{i}\right)$, raw point cloud as $P_{\mathrm{s}}$, and $P_{\mathrm{s}}=$ $\left\{P_{i} \in R^{3} \mid P_{0}, P_{1}, P_{2}, \ldots, P_{n}\right\}$. The transformation of the point cloud coordinate system is shown in Fig. 9.

Here, $O_{\mathrm{s}}$ is the coordinate system of the 3D scanner, and $O_{\mathrm{m}}$ is the coordinate system of machining. As shown in

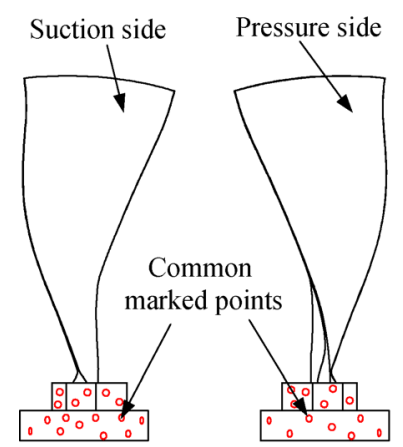

(a) (b)



(c)
Fig. 8 Scanning the blade, fixture and chuck as a whole. (a) One side of the parts; (b) another side of the parts; (c) registration of the point cloud of the parts.

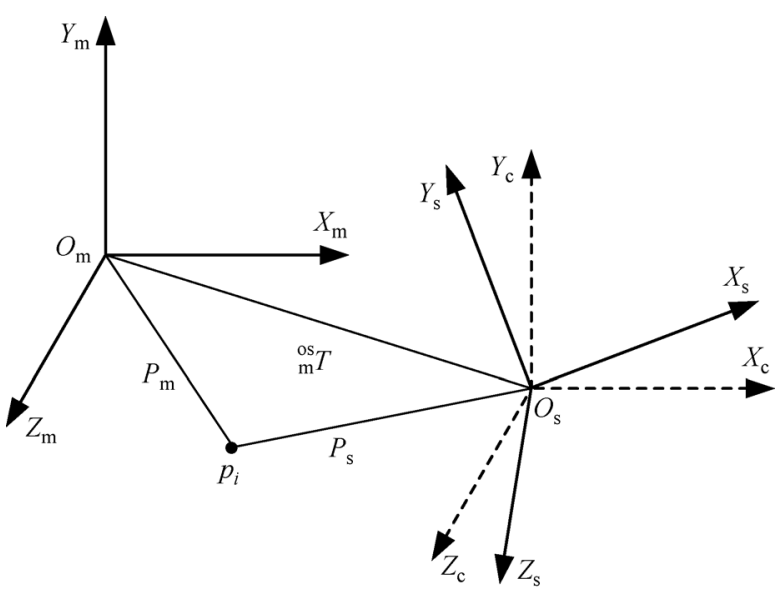

Fig. 9 Transformation of the point cloud coordinate system.

Eq. (1), the point cloud based on the 3D scanner coordinate system can be converted into the machining coordinate system.

$$
P_{\mathrm{m}}={ }_{\mathrm{m}}^{\mathrm{s}} R P_{\mathrm{s}}+{ }_{\mathrm{m}}^{\mathrm{s}} T,
$$

where $P_{\mathrm{m}}$ denotes the point based on the machining coordinate system, $P_{\mathrm{s}}$ is the point based on the $3 \mathrm{D}$ scanner, ${ }_{\mathrm{m}}^{\mathrm{s}} R P_{\mathrm{s}}$ represents the rotation transformation from $P_{\mathrm{s}}$ to $P_{\mathrm{m}}$, where ${ }_{\mathrm{m}}^{\mathrm{s}} R$ is the rotation matrix, and ${ }_{\mathrm{m}}^{\mathrm{s}} T$ is the translation matrix from $P_{\mathrm{s}}$ to $P_{\mathrm{m}}$.

Noise points are inevitably introduced into the raw point cloud because of many factors, such as the accuracy of the measurement device, scanning speed, and surface quality of measured parts and environment. These noise points seriously affect the accuracy of the measured workpiece and should thus be removed. Generally, point cloud denoising algorithms include least squares, smooth, and adaptive bilateral filtering [31]. Initially, Euclidean cluster extraction is applied to remove the noises outside the object. In consideration of the curvature that changes 
significantly at the LE and TE of the blade, an adaptive bilateral filtering algorithm is adopted to remove the noise points mixed in the target point cloud to retain many edge features of the blade. However, there are still some missing points at the LE and TE of the blade, as shown in Fig. 10(a). Thus, a hole filling method based on moving least squares is adopted [32]. This method first identifies the hole boundary, then its vicinity is used to interpolate the missing portion by using moving least squares, as shown in Fig. 10(b).

Numerous redundant points exist in the raw point cloud, thus delaying the processing and affecting the smoothness of the point cloud. Thus, many raw point clouds should be reduced. To maintain the shape characteristics of an aeroengine blade, voxelized grid reduction is used to reduce the number of point cloud data. Point cloud processing is shown in Fig. 11. The part in the red box is the target point cloud. Numerous noise points exist inside or outside the target point cloud. First, Euclidean cluster extraction and adaptive bilateral filter algorithm are used to remove the noise points from the raw point cloud data. Then, voxelized grid point cloud reduction is used to obtain the ideal point cloud data.

\subsection{Automatic extraction of point cloud}

The regions that need to be strengthened are generally the blade root, the blade near the edge, and the local region of the damaged blade after laser repair. Thus, not all surfaces of the blade should be strengthened. The local region that needs to be strengthened from the point cloud of the blade should be extracted. Although some software, such as Imageware, Geometric Studio, and CloudCompare, can manually extract or delete a certain shape of point cloud, they cannot accurately extract a point cloud with a specific size from scattered point cloud data. This study uses a local point cloud automatic extraction algorithm to search the entire blade point cloud globally. The blade edge and the coordinate of the to-be-strengthened region are used to extract the point cloud of the local region of the blade automatically.

The local region on one side of the blade surface between $Z_{a} \leqslant Z \leqslant Z_{b}$ is extracted, as shown in Fig. 12. The main steps of extraction are expressed as follows:

Step 1: All point clouds of the blade in interval $Z_{a} \leqslant Z$ $\leqslant Z_{b}$ are extracted.

Step 2: The edge point cloud data on the two sides of the interval are removed. The LE and TE of the blade cannot be strengthened to the edge of the blade because they are the arc transition area, and the curvature changes considerably. First, the point cloud in the region is stratified in the $Z$ direction. Second, all points for each layer of the point cloud are extracted within the range $\min (Y)+\Delta \leqslant Y \leqslant \max (Y)-\Delta$, where $\Delta$ denotes the offset distance. This process obtains the two-sided point cloud of the blade within the specific region.

Step 3: One side of the point cloud data of a specific region is extracted. First, the two-sided point cloud is stratified in the $Y$ direction, and the thickness of each layer is denoted by $\varepsilon$. Second, suppose $p_{i, j}\left(x_{i, j}, y_{i, j}, z_{i, j}\right)$ is a point on layer $j$, and $S_{j}(x)$ is a specific plane on layer $j$, as shown in Eq. (2), and $d_{i, j}$ is the distance from point $p_{i, j}\left(x_{i, j}, y_{i, j}\right.$, $\left.z_{i, j}\right)$ to specific plane $S_{j}(x)$, which is calculated using Eq. (3). Corresponding point $p_{i, j}$ belongs to the selected region when Eq. (4) is satisfied.

$$
\begin{gathered}
S_{j}(x)=\max \left(X_{j}\right)+\mathrm{C}, \\
d_{i, j}=p_{i, j}-S_{j}(x)=\left|x_{i, j}-\max \left(X_{j}\right)-\mathrm{C}\right|, \\
d_{i, j} \leqslant \frac{d_{\text {max }, j}+d_{\text {min }, j_{j}}}{2},
\end{gathered}
$$

where $X_{j}$ is the set of the $x$-coordinate of all points in layer

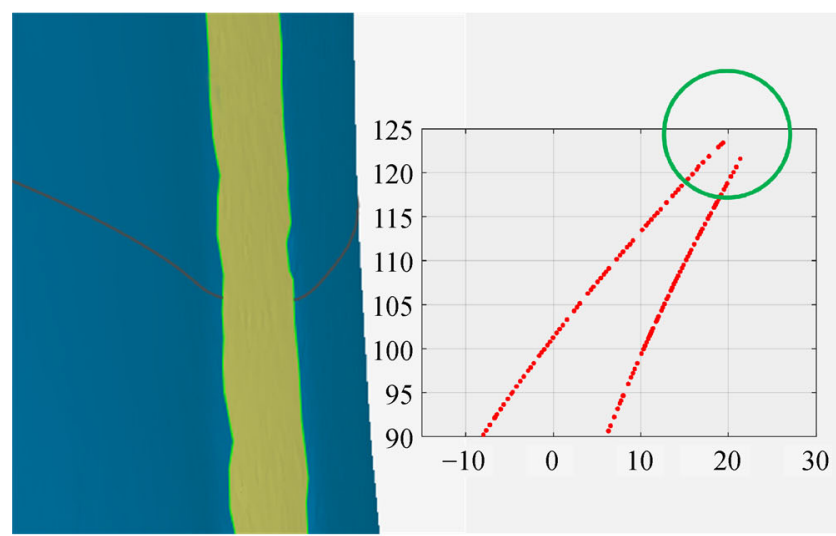

(a)
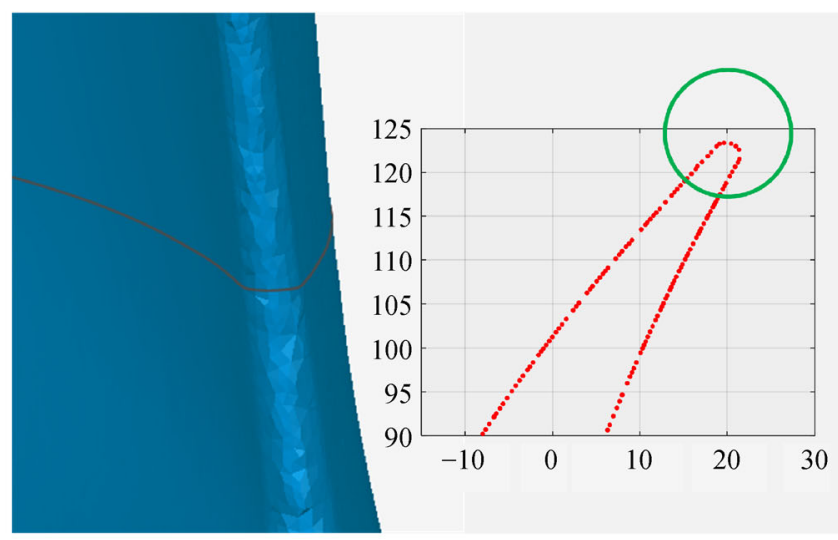

(b)

Fig. 10 Processing of the LE and TE. (a) Missing portion of the blade edge; (b) blade edge after hole filling. 


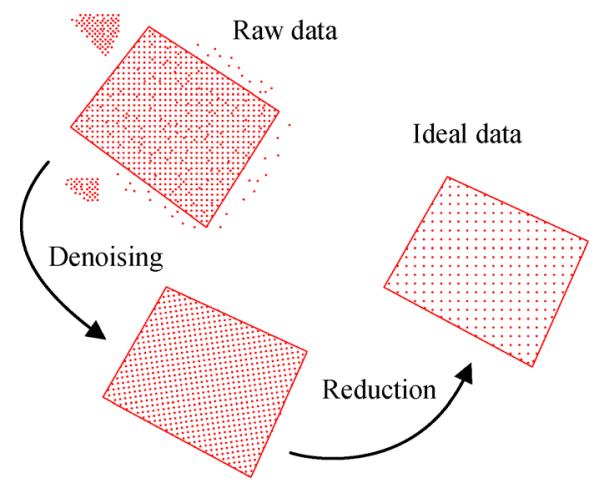

Fig. 11 Point cloud processing.

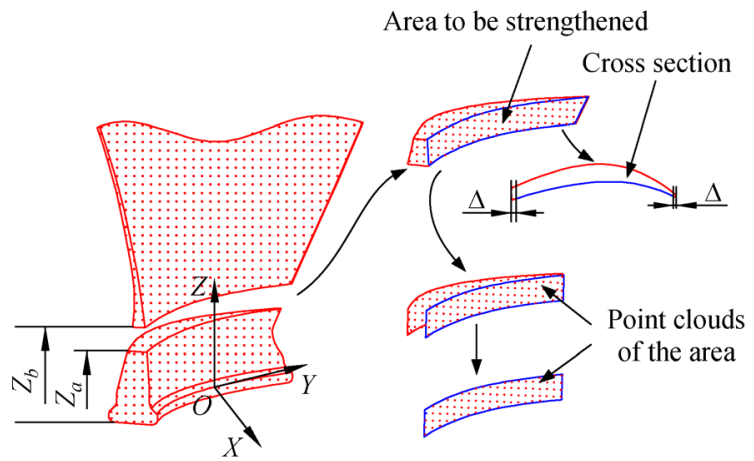

Fig. 12 Extraction of the blade local region point cloud.

$j, \mathrm{C}$ is a constant larger than $0, d_{\max , j}$ and $d_{\min , j}$ are the maximum and minimum distances from the point to plane $S_{j}(x)$ on layer $j$, respectively.

Suppose that the local region that needs to be strengthened is a circular area on the blade, the center of the circular area is at point $P_{\mathrm{o}}\left(x_{\mathrm{o}}, y_{\mathrm{o}}, z_{\mathrm{o}}\right)$, and its radius is $R$. The extraction process is shown in Fig. 13. The steps are explained as follows:

Step 1: All point cloud data within interval $D \leqslant R$ are extracted, where $D$ is the distance from all blade point

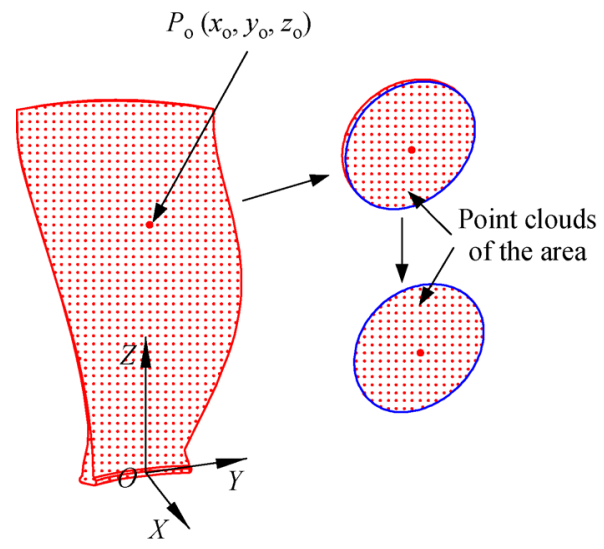

Fig. 13 Extraction of the circular area point cloud. clouds $P_{i}=\left(x_{i}, y_{i}, z_{i}\right)$ to a specific point $P_{\mathrm{o}}$, which is calculated using Eq. (5). This process obtains the twosided point cloud of the circular area.

$$
D=\sqrt{\left(x_{i}-x_{\mathrm{o}}\right)^{2}+\left(y_{i}-y_{\mathrm{o}}\right)^{2}+\left(z_{i}-z_{\mathrm{o}}\right)^{2}} .
$$

Step 2: This step extracts one side point cloud of the circular area. The method is the same as that in Step 3.

The border of the strengthening region is guaranteed by the blade edge and specific coordinates. The local point cloud automatic extraction algorithm can accurately extract the point cloud data of any region to be strengthened, thus improving the accuracy and efficiency of blade strengthening.

\section{TS-USRP machine tool path generation}

TS-USRP machining tool path planning for aeroengine blades mainly includes determining the tool movement mode, selecting the appropriate step length and row spacing of the path, fitting the extracted point cloud of the region to be strengthened, and generating the TS-USRP machining tool path of the blade.

Figure 14 shows the two tool motion modes of the blade, namely, transverse and longitudinal motions. Although the amount of tool reversing is less than the longitudinal motion, the single path is longer and causes a large accumulated error. Conversely, the single path of the transverse motion is short, and the accumulated error is small. The transverse motion is suitable for machine control and blade machining based on the blade structure and the developed TS-USRP machine tool.

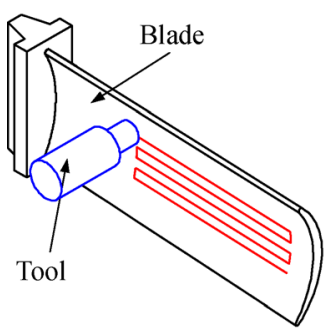

(a)

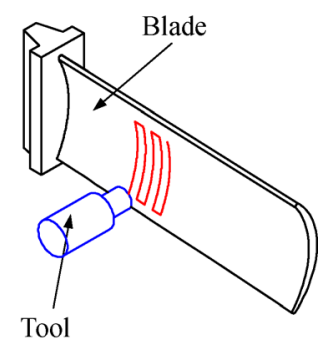

(b)
Fig. 14 Tool motion modes. (a) Longitudinal motion; (b) transverse motion.

Step length, which is denoted by $s$, is the distance between two adjacent tool points on the same path. $l$ is the row spacing of the path, which is the distance between two adjacent paths. In $\mathrm{NC}$ machining, the tool cannot completely move along the designed curve, and the designed curve can be gradually approached by the broken line trajectory, inevitably resulting in a certain 
approximation error. A small step length corresponds to a small approximation error and low processing efficiency.

\subsection{Point cloud fitting and path planning}

Point cloud fitting and path planning are shown in Fig. 15. Figure 15(a) shows the local point cloud extracted from the processed blade point cloud. First, the point cloud is projected along the $X$-axis to the $Y Z$ plane. The quadrangle boundary along the $Y$ and $Z$ directions is obtained, that is, $y_{\max }, y_{\min }, z_{\max }$, and $z_{\min }$ are calculated. Thus, the quadrangle is a rectangle with side length $\left(y_{\max }-y_{\min }\right) \times$ $\left(z_{\max }-z_{\min }\right)$. Second, the quadrangle is divided into grids in terms of step length and row spacing of the desired machining path, and the number of grids along the $Y$ and $Z$ directions is $n_{y}$ and $n_{z}$, respectively. $n_{y}$ and $n_{z}$ are calculated as follows:

$$
\begin{aligned}
& n_{y}=\frac{y_{\max }-y_{\min }}{s}, \\
& n_{z}=\frac{z_{\max }-z_{\min }}{l},
\end{aligned}
$$

where $s$ denotes the step length of the desired machining

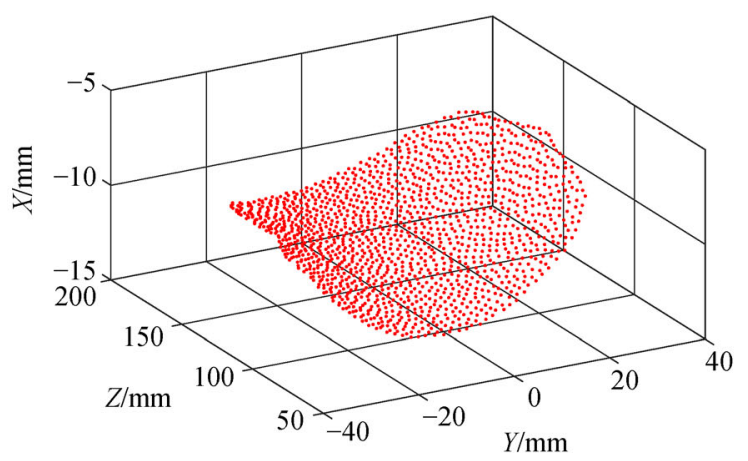

(a)

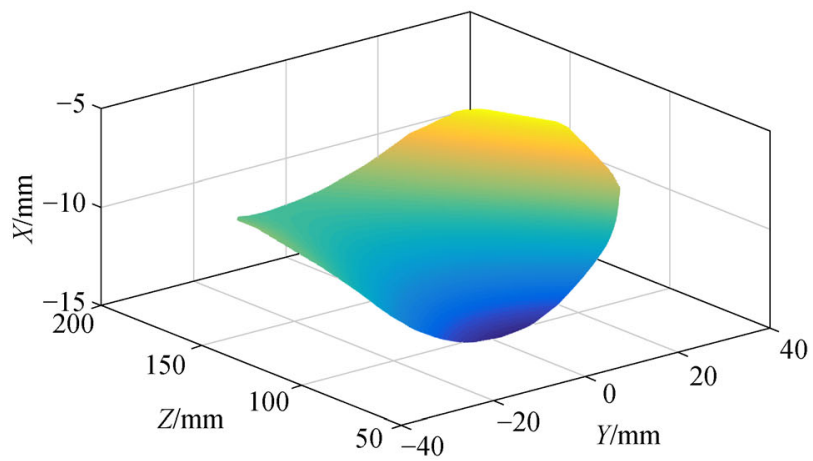

(c) path, and $l$ denotes the row spacing. Each grid point is denoted as $\left(y_{i}, z_{j}\right)$, where $i=1,2, \ldots, n_{y}+1, j=1,2, \ldots$, $n_{z}+1$, as shown in Fig. 15(b).

The 2D linear interpolation approach is applied to fit the points on grid $(y, z)$. Suppose $x_{k}\left(y_{k}, z_{k}\right), k=1,2,3,4$ are the four nearest points of point $x(y, z)$ in four different quadrants, $x(y, z)$ can be expressed as

$$
x(y, z)=\sum_{k=1}^{4} x_{k}\left(y_{k}, z_{k}\right)\left(y_{k}-y\right)\left(z_{k}-z\right) .
$$

Thus, the mesh surface of the blade's local region to be strengthened, and its corresponding grid points $(y, z, x)$ are obtained, as shown in Fig. 15(c).

This study adopts a transverse zigzag-motion mode for path planning on the basis of the above fitting and interpolation results of the point cloud, and the cutter contact (CC) points on one side of the local region are obtained, as shown in Fig. 15(d).

\subsection{Machine tool path generation}

The TS-USRP machine tool path should be calculated on the basis of the CC point to achieve two-sided blade

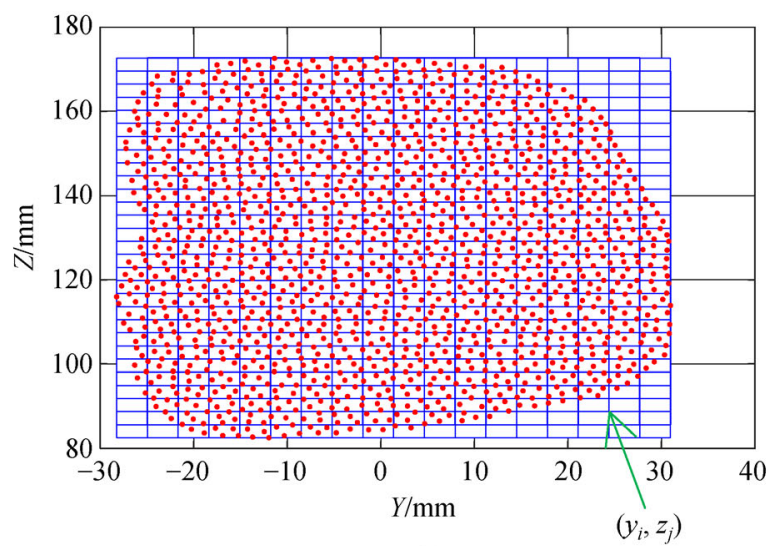

(b)

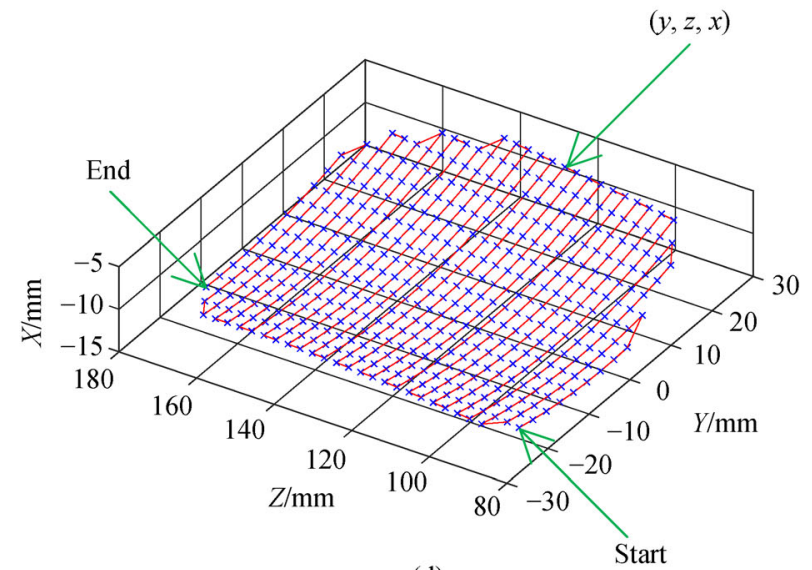

(d)

Fig. 15 Point cloud fitting and path planning. (a) Local region point cloud; (b) point cloud projection and meshing; (c) point cloud fitting; (d) path planning of the local region. 
strengthening. The principle of the TS-USRP machine tool system is shown in Fig. 16. The required input path of this system is denoted as $P^{\prime}[x, y, z, \omega, d]$, where $[x, y, z]$ is the $\mathrm{CC}$ point of one side of the region to be strengthened, $\omega$ is the rotation angle of the blade, and $d$ is the thickness of the blade at the corresponding CC point. Thus, the rotation angle and thickness are calculated.

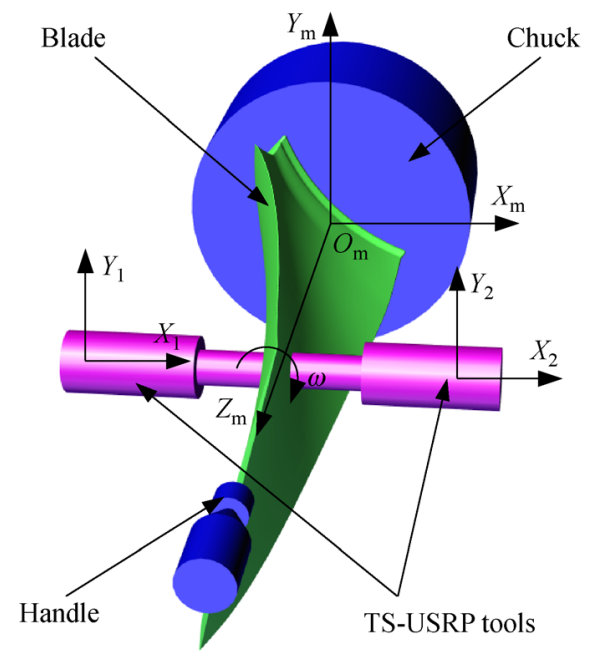

Fig. 16 Principle of a TS-USRP machine tool system.

TS-USRP tools should remain perpendicular to the blade surface during processing. In this study, the rolling head is perpendicular to the line connecting the interpolation points to approximate its perpendicularity to the blade surface. Hence, the blade should be continuously rotated to adjust the posture during machining, as shown in Fig. 17. The blade rotation angle is calculated as follows: Suppose $a, b$ and $c$ are $\mathrm{CC}$ points, and $\beta$ is the angle between line $a b$ and the $Y$-axis direction. Blade rotation angle $\omega$ is equal to $\beta$. The slope of line $a b$ is denoted as $k_{a b}$. Angle $\beta$ is determined as

$$
\beta=\frac{\pi}{2}-\left|\arctan k_{a b}\right|
$$

Thus, the blade rotation angle is expressed as

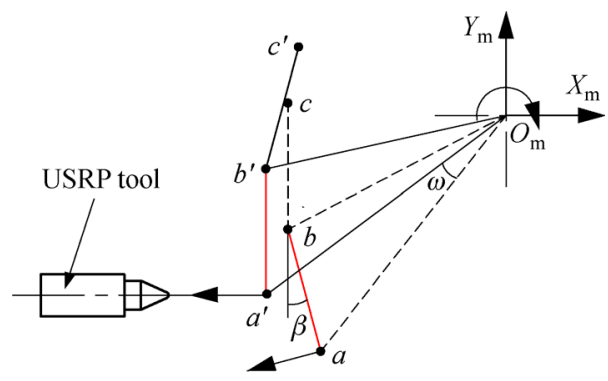

Fig. 17 Calculation of blade rotation angle.

$$
\omega_{i}= \begin{cases}\frac{\pi}{2}-\arctan \left(k_{i, i+1}\right), & k_{i, i+1}>0, \\ \frac{\pi}{2}-\left|\arctan \left(k_{i, i+1}\right)\right|, & k_{i, i+1} \leqslant 0,\end{cases}
$$

where $k_{i, i+1}=\frac{y_{i+1}-y_{i}}{x_{i+1}-x_{i}}$.

Subsequently, the blade thickness at the corresponding $\mathrm{CC}$ point is calculated as follows: As shown in Fig. 18, $p_{i}$ and $p_{i+1}$ are denoted as two adjacent points of the CC path. Theoretically, the USRP tool is along the normal direction of point $p_{i}$ on the CC path. In this study, the direction of line segment $p_{i} p_{i+1}$ is approximated to indicate the tangential direction of the CC path at point $p_{i}$. Thus, the USRP tool is perpendicular to line segment $p_{i} p_{i+1}$. Line $l_{i}$, which goes through $p_{i}$, is perpendicular to $p_{i} p_{i+1}$. Then, intersection point $p_{i}^{\prime}$ between line $l_{i}$ and surface $s(u, v)$ formed by the point cloud of the other side of the blade can be obtained. Blade thickness $d$ corresponding to point $p_{i}$ is calculated as

$$
d=p_{i}-p_{i}^{\prime}
$$

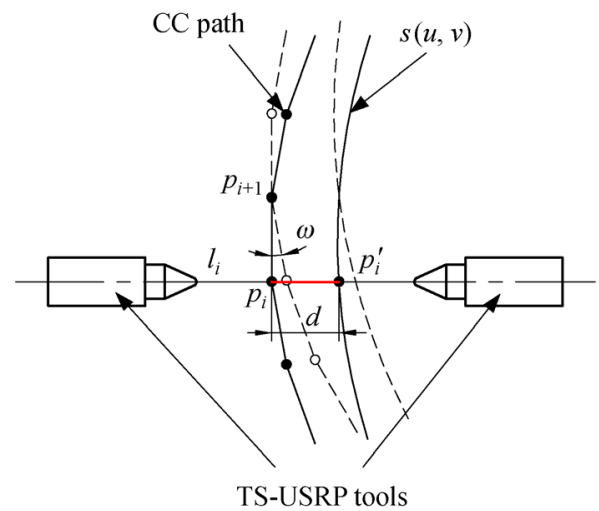

Fig. 18 Calculation of thickness.

As previously discussed, $P^{\prime}[x, y, z, \omega, d]$ is the required input path of the TS-USRP machine tool system. Thus, the input path should be converted into the control quantity of the system. As shown in Fig. 16, the control quantity of the system is denoted as $P\left[x_{1}, y_{1}, x_{2}, y_{2}, z, \omega\right]$, where $x_{1}$ is the horizontal displacement of the left side of the USRP tool; $x_{2}$ is the horizontal displacement of the other side of the USRP tool; $y_{1}$ and $y_{2}$ are the vertical displacements of the USRP tool, respectively; $z$ is the blade feed along the $Z$-axis; and $\omega$ is the blade rotation angle. The transformation of $P^{\prime}[x, y, z, \omega, d]$ and $P\left[x_{1}, y_{1}, x_{2}\right.$, $\left.y_{2}, z, \omega\right]$ is expressed as follows:

$$
P\left[x_{1}, y_{1}, x_{2}, y_{2}, z, \omega\right]=P^{\prime}[x, y, z, \omega, d] \cdot \boldsymbol{n},
$$

where $\boldsymbol{n}$ is the transfer matrix, as described below: 


$$
\boldsymbol{n}=\left[\begin{array}{cccccc}
\cos \omega & \sin \omega & \cos \omega & \sin \omega & 0 & 0 \\
-\sin \omega & \cos \omega & -\sin \omega & \cos \omega & 0 & 0 \\
0 & 0 & 0 & 0 & 1 & 0 \\
0 & 0 & 0 & 0 & 0 & 1 \\
0 & 0 & 1 & 0 & 0 & 0
\end{array}\right]
$$

\section{Experiment}

The equipment of the blade TS-USRP machine tool is developed and used to conduct the experiments. It consists of control, cooling, clamping, and strengthening systems, as shown in Fig. 19. The special fixture of the blade is shown in Fig. 20.

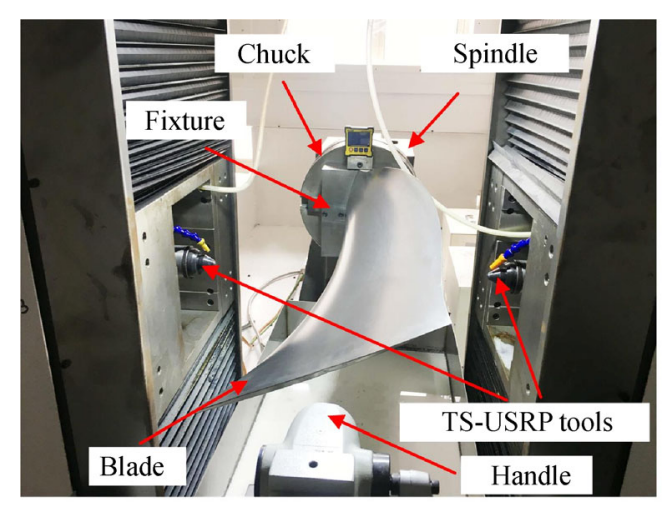

Fig. 19 Equipment of the blade TS-USRP machine tool.



Fig. 20 Special fixture of the blade.

\subsection{Experimental setup}

1) OMNCM pretreatment. The noncontact measuring system adopts an HL-3DS+ structured light-based 3D scanner. The scanner settings and the incidence angle of structure light can affect the scanning accuracy. For the aeroengine blade, the small scan mode with a single scan range of $200 \mathrm{~mm} \times 200 \mathrm{~mm}$ and single scanning accuracy of $\pm 0.006 \mathrm{~mm}$ is selected, and the scanning distance is within $630 \mathrm{~mm}$. The incidence angle of the structure light is adjusted to ensure that the scanner can identify at least three marked points. According to experience, the scanner can identify the marked points when the incident angle of the scanner is within $30^{\circ}$. As shown in Fig. 21, a calibration board is used for 3D scanner calibration before measurement to ensure the accuracy of the measured workpiece. Surface reflection can seriously affect the scanning accuracy because of the metallic luster on the blade surface. Thus, developer should be sprayed on the measured workpiece surface. The marked point is pasted for point cloud registration, as shown in Fig. 22.

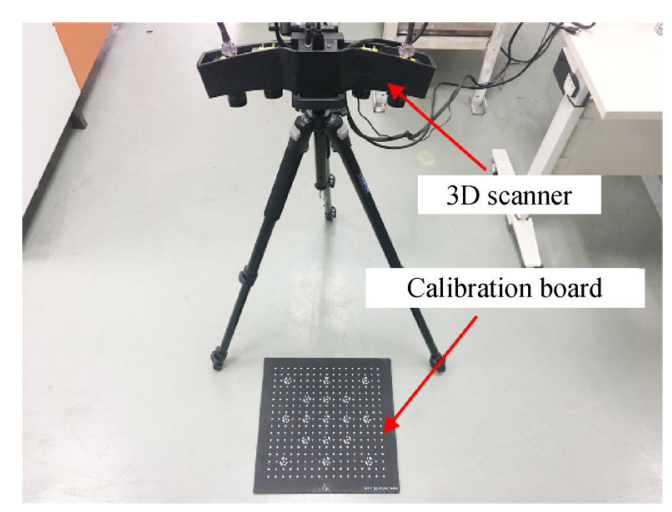

Fig. 21 Calibration of the 3D scanner.

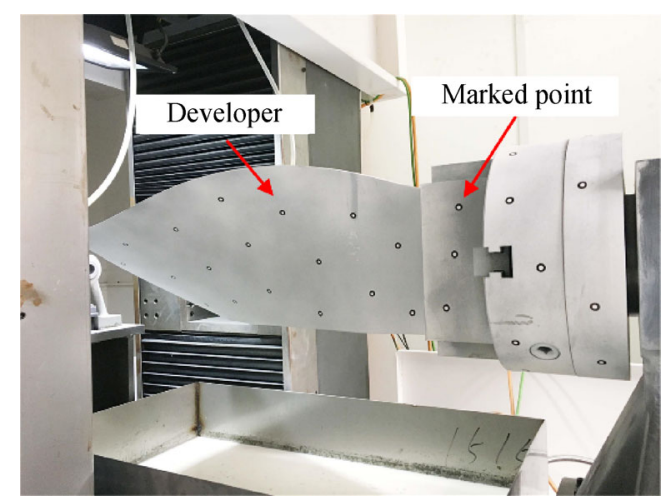

Fig. 22 Spraying developer and pasting marked points.

2) Blade OMNCM. Figure 23 shows the OMNCM process. The blade is rotated by controlling the spindle of the machine tool during scanning. The point cloud is registered using the marked points on the chuck and fixture. As the curvature of the blade changes greatly, the change of curvature will significantly affect the scanning accuracy. Therefore, this experiment adopts multi-angle and multi-amplitude matching to ensure scanning 


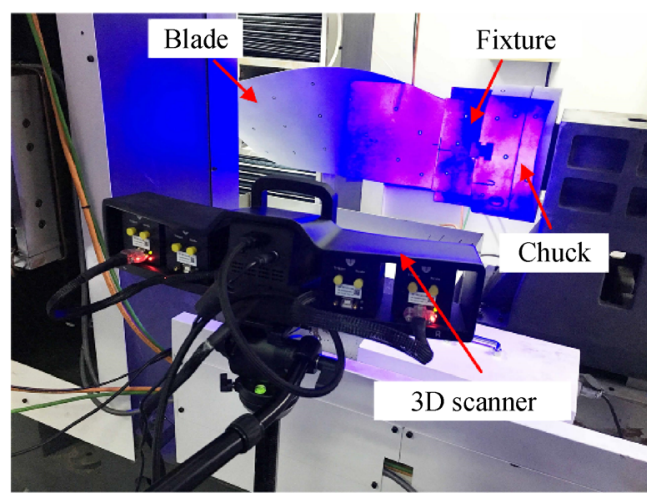

Fig. 23 OMNCM process.

accuracy. The raw point cloud, which includes the blade point cloud, chuck, and fixture with a total of 9129911 data points, is shown in Fig. 24.

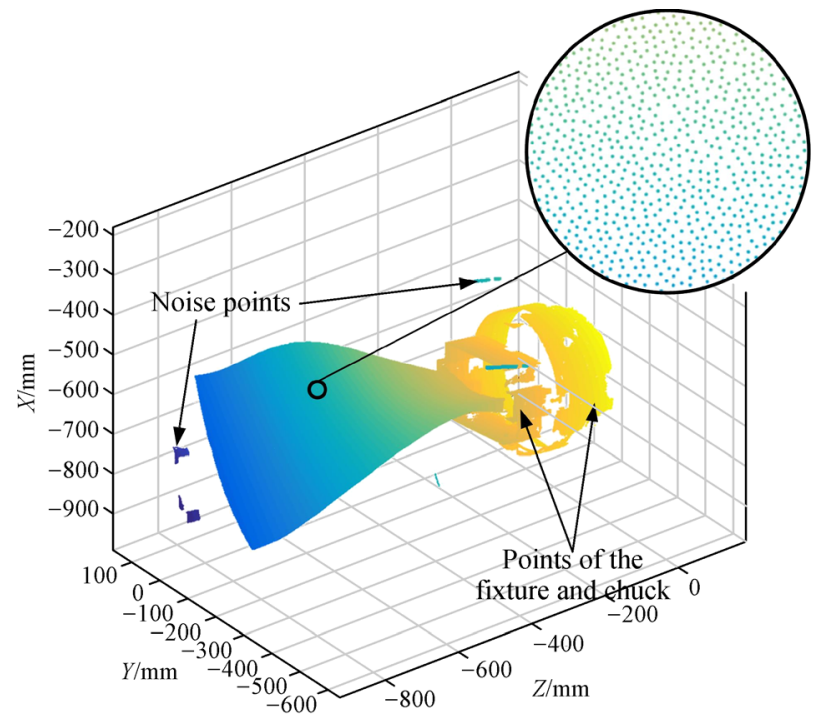

Fig. 24 Raw point cloud obtained through OMNCM.

3) Point cloud processing. As shown in Fig. 24, many noise and redundant points are mixed in the raw point cloud. Hence, point cloud processing is crucial for subsequent tool path planning. First, Euclidean cluster extraction algorithm is used to remove the noises outside the object. Then, an adaptive bilateral filtering algorithm is adopted to remove the noise points mixed in the point cloud of the blade body. The blade point cloud is obtained through point cloud coordinate conversion, denoising, point cloud reduction, and removal of the point cloud of the chuck and fixture (Fig. 25).

4) Measurement accuracy detection. As mentioned above, the profile of the nominal blade is accurate. In this paper, the Geomagic Control software is used to best fit align the measured blade profile with the nominal blade, as shown in Fig. 26. The number of data points of the

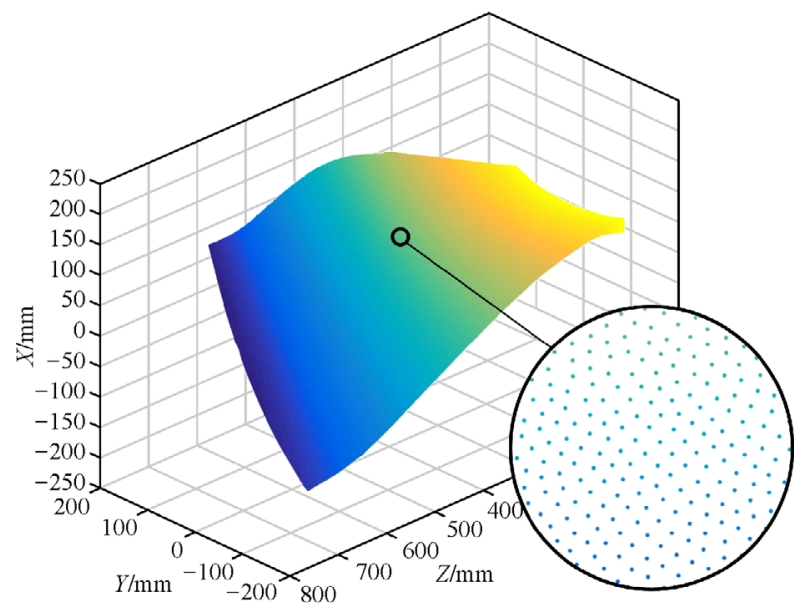

Fig. 25 Processed point cloud of the blade.

processed measured blade profile is 1034121. Table 1 shows the distribution of deviations between measured points and nominal blade profile. The points with a deviation within $(-1.7850,1.7850) \mathrm{mm}$ account for $99.8744 \%$ of the total measured points. Table 2 shows the best fit alignment results with an average deviation within $(-0.1954,0.1954) \mathrm{mm}$ and standard deviation of $0.3321 \mathrm{~mm}$, thus indicating that the measured blade has high accuracy.

\subsection{TS-USRP machining on the blade}

As mentioned above, the vulnerable areas of the blade include the root of the blade and the area near the blade edge. The laser-repaired area also needs to be strengthened. Therefore, an experiment is conducted on the blade root, the area near the blade edge, and the local area of the blade body.

As shown in Fig. 27(a), a rectangular region in the middle of the blade is selected as the area to be strengthened. The height of the rectangle is 300 $\mathrm{mm} \leqslant Z \leqslant 320 \mathrm{~mm}$, and the width is $80 \mathrm{~mm}$. The point cloud of the local region is extracted from the blade point cloud by using the proposed automatic extraction algorithm, as shown in Fig. 27(b). The experimental step length and row spacing of the path are $0.1 \mathrm{~mm}$. Subsequently, point cloud fitting and path planning of the region to be strengthened are conducted, as shown in Figs. 27(c) and 27(d). Then, the TS-USRP machine tool path is obtained when the blade thickness at the corresponding $\mathrm{CC}$ point and the blade rotation angle are calculated. The local region of the blade is strengthened by the blade TS-USRP machine tool system, as shown in Fig. 27(e). During strengthening, the ultrasonic heads move smoothly along the planned path and remain in contact with the blade. Figure 27(f) shows the result of TS-USRP strengthening of the local region. The strengthening process in this local region takes a total of $22 \mathrm{~min}$. 


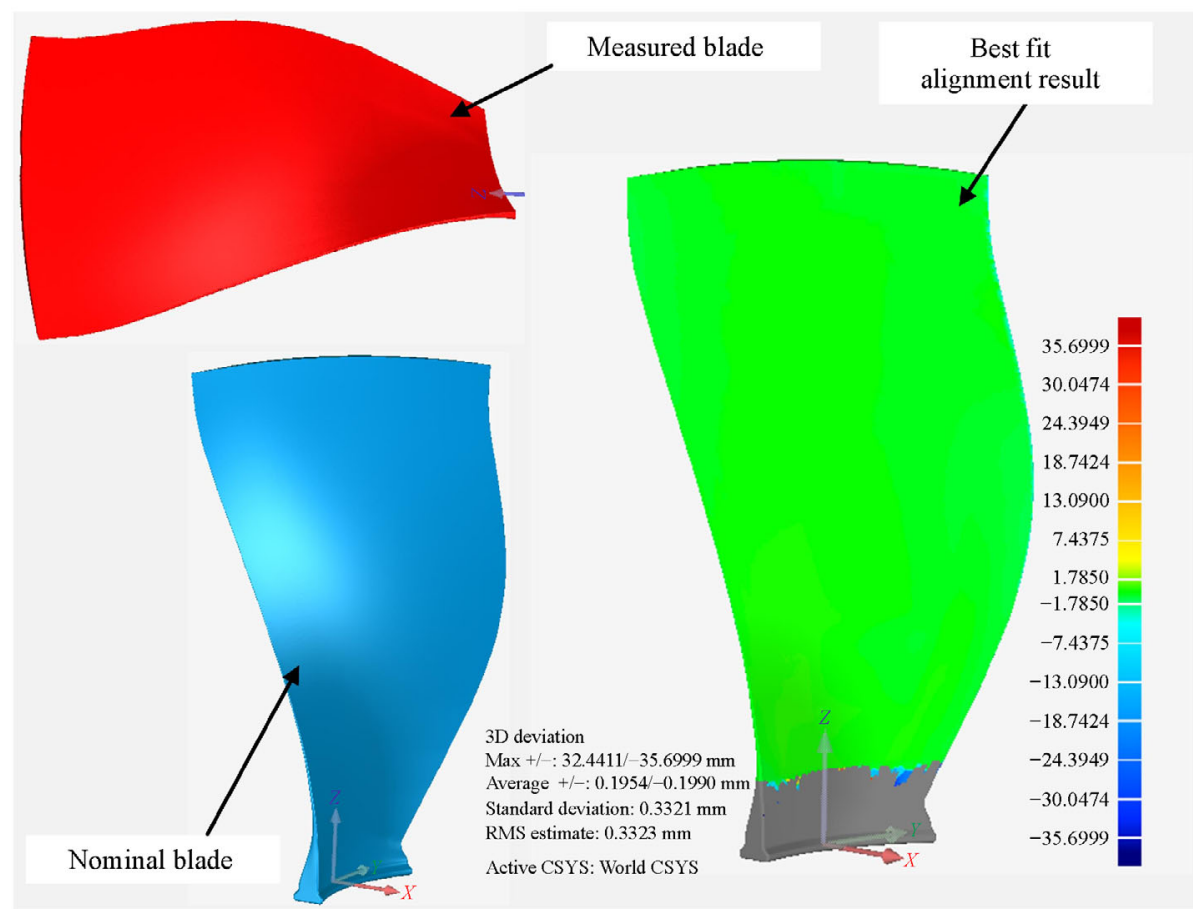

Fig. 26 Best fit alignment of the measured blade profile and nominal blade.

Table 1 Deviation distribution of the best fit alignment

\begin{tabular}{|c|c|c|c|}
\hline \multicolumn{2}{|c|}{ Deviation range/mm } & \multirow{2}{*}{ Number of points } & \multirow{2}{*}{ Proportion/\% } \\
\hline$\geqslant \operatorname{Min}$ & $<\operatorname{Max}$ & & \\
\hline-35.6999 & -30.0474 & 7 & 0.0007 \\
\hline-30.0474 & -24.3949 & 14 & 0.0014 \\
\hline-24.3949 & -18.7424 & 23 & 0.0022 \\
\hline-18.7424 & -13.0900 & 12 & 0.0012 \\
\hline-13.0900 & -7.4375 & 19 & 0.0018 \\
\hline-7.4375 & -1.7850 & 1215 & 0.1175 \\
\hline-1.7850 & 1.7850 & 1032822 & 99.8744 \\
\hline 1.7850 & 7.4375 & 6 & 0.0006 \\
\hline 7.4375 & 13.0900 & 1 & 0.0001 \\
\hline 13.0900 & 18.7424 & 0 & 0.0000 \\
\hline 18.7424 & 24.3949 & 0 & 0.0000 \\
\hline 24.3949 & 30.0474 & 0 & 0.0000 \\
\hline 30.0474 & 35.6999 & 2 & 0.0002 \\
\hline
\end{tabular}

Similarly, the blade root area (range from $Z=80 \mathrm{~mm}$ to $Z=100 \mathrm{~mm}$ ) and the area near the blade edge (range from $Z=100 \mathrm{~mm}$ to $Z=650 \mathrm{~mm}$ with a width of $20 \mathrm{~mm}$ ) are strengthened, as shown in Fig. 28. The experimental results prove that the proposed method is effective and efficient.

\subsection{Measurement}

The blade surface roughness was measured before and after the experiment by using a TR220 digital surface roughness meter. Figure 29 shows the surface roughness measurement method. Nos. 1-12 are the test positions of the strengthened region. Five points are taken from each position for measurement, and the average roughness of the position is calculated. Figure 30 shows the measurement result of surface roughness before and after the strengthening, and the blue part is the blade surface roughness without TS-USRP strengthening. The experiment results demonstrate that the proposed methods can reduce the surface roughness and achieve uniform roughness of aeroengine blades. 
Table 2 Best fit alignment results

\begin{tabular}{lc}
\hline Best fit alignment deviation index & Deviation $/ \mathrm{mm}$ \\
\hline Max. upper deviation & 32.4411 \\
Max. lower deviation & -35.6999 \\
Average deviation & $(-0.1954,0.1954)$ \\
Standard deviation & 0.3321 \\
\hline
\end{tabular}

To verify the machining accuracy of the proposed method, the geometric accuracy of the strengthened region is measured by using computer vision techniques. Figure 31 shows the strengthened regions processed with and without using the proposed method. The distance between the strengthened region edge and the blade edge at the coordinate range of $Z=100$ to $Z=-200 \mathrm{~mm}$ is measured. Within this range, 10 corresponding measurement positions were taken for each strengthening result, five measurements were performed at each position, and the final measurement results were averaged. The theoretical result based on the blade model is $0.5 \mathrm{~mm}$. However, the geometric accuracy of the processing is much lower due to the blade model error and fixing error. As a result, the actual distance is about $2.83 \mathrm{~mm}$ on average, a value that can be improved to $0.79 \mathrm{~mm}$ with the use of the proposed method. The experiment demonstrates that the proposed method can effectively reduce the processing errors caused by models and clamping, thereby improving the processing accuracy.

\section{Conclusion and future work}

Aeroengine blades are thin-walled parts with a free-form surface and limited life. Thus, this study presents a TSUSRP technique for blade strengthening and develops a blade TS-USRP machine tool system. OMNCM is used due to the deviation between the actual and nominal blades and the clamping error in secondary machining. Point cloud processing is conducted, and a local point cloud

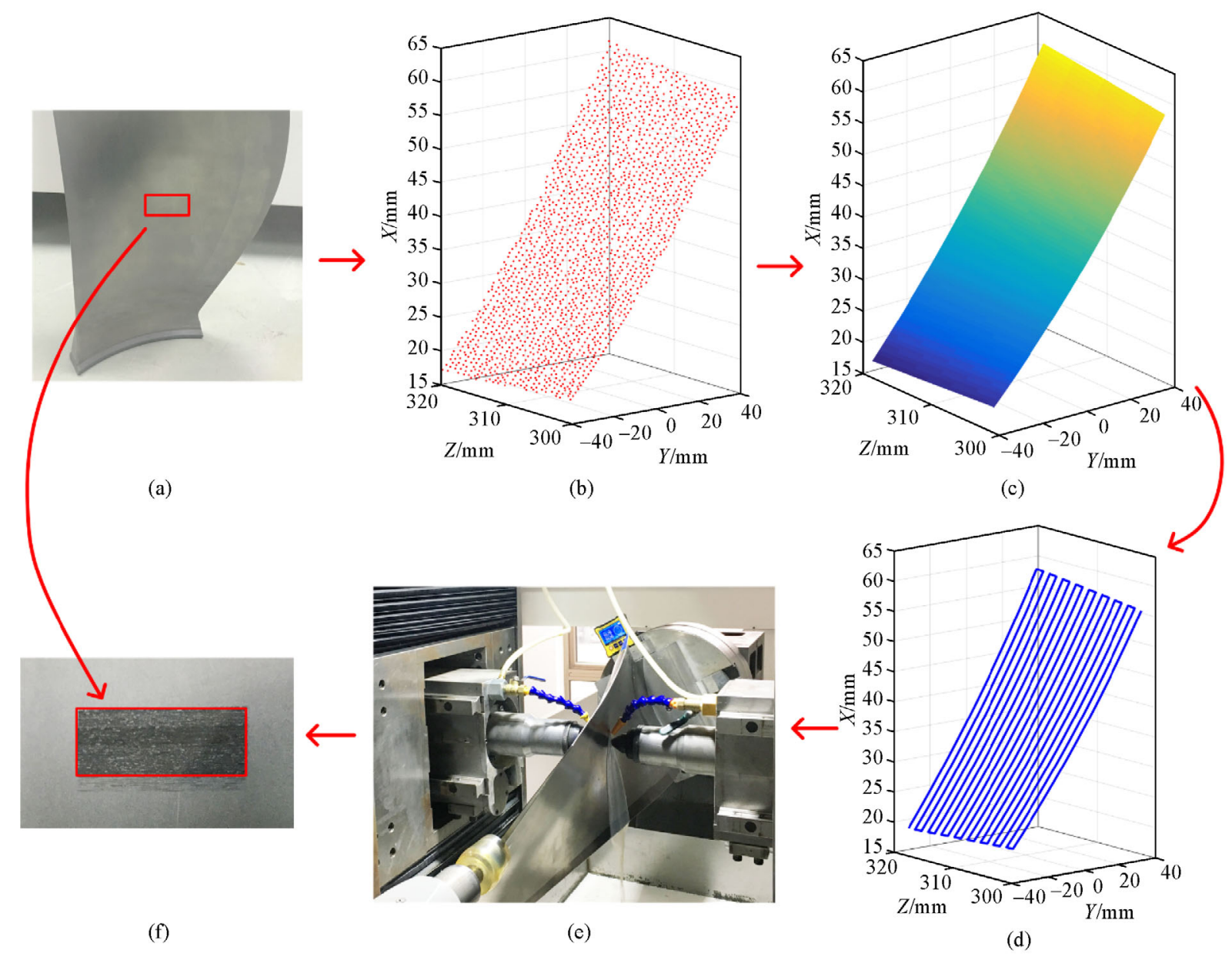

Fig. 27 TS-USRP strengthening of the blade local region. (a) Local region to be strengthened; (b) point cloud of the local region; (c) point cloud fitting; (d) machining path planning; (e) TS-USRP machining of the local region; (f) local region after TS-USRP strengthening. 


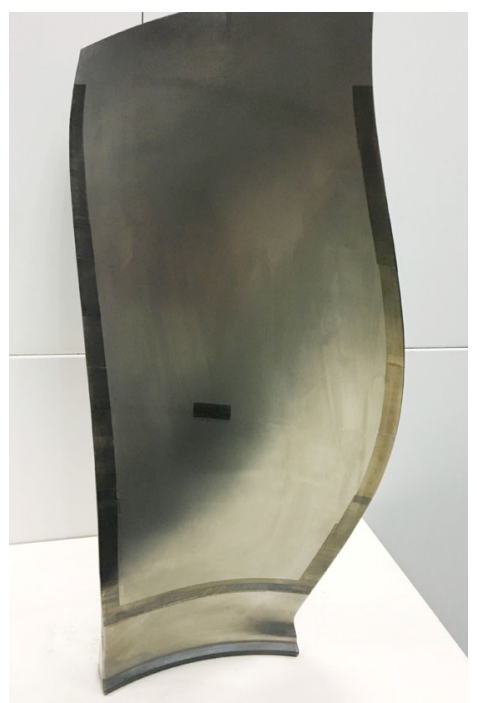

Fig. 28 Result of the strengthened blade.

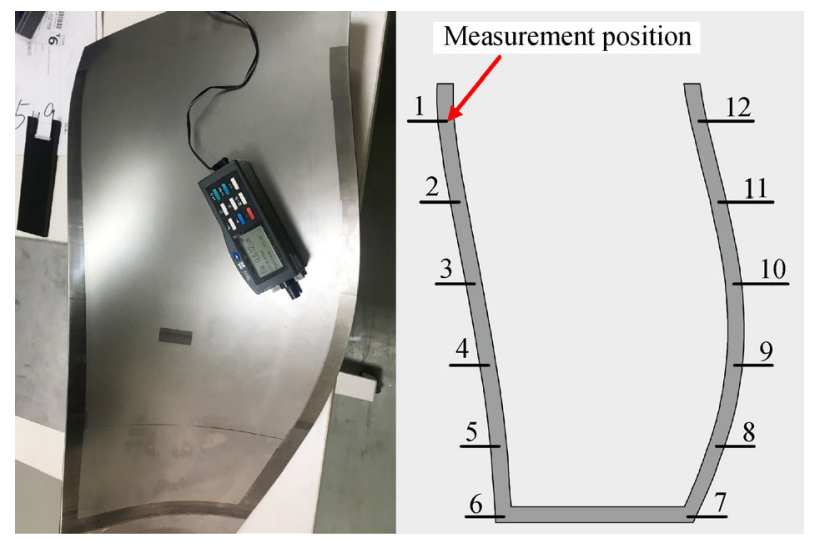

Fig. 29 Surface roughness measurement.

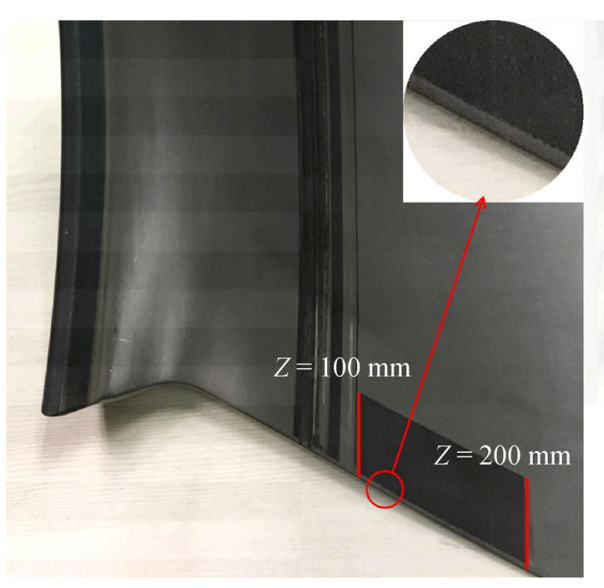

(a)

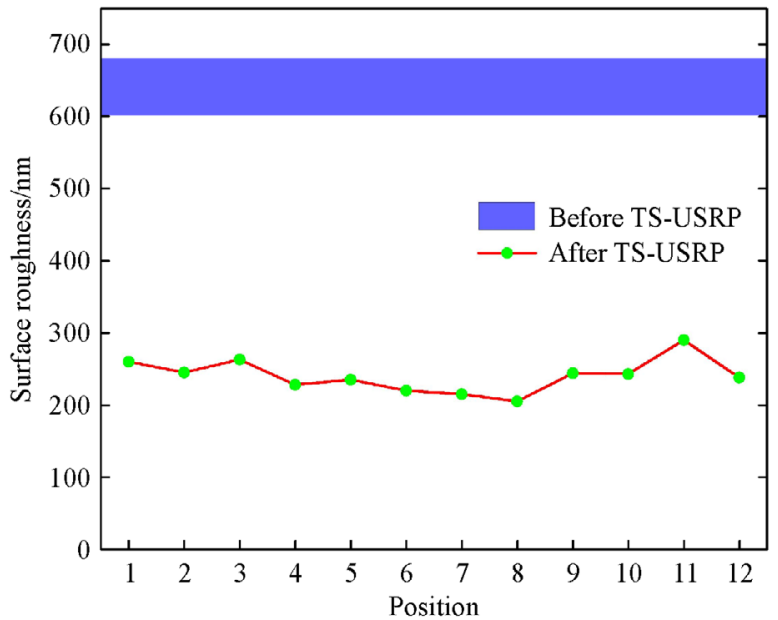

Fig. 30 Surface roughness of before and after the strengthening.

automatic extraction algorithm is presented to improve the accuracy and efficiency of blade strengthening. The blade local region is strengthened, and the proposed methods are verified through an experiment. Results prove the effectiveness of the developed blade TS-USRP strengthening system and indicate that OMNCM and a series of point cloud processing steps for the blade are effective. The proposed method can be applied to strengthen the region where the blade is susceptible to damage and the local region of the damaged blade after laser cladding repair.

However, adjusting the ultrasonic generator on both sides to the same frequency and amplitude cannot guarantee the synchronized vibration of the TS-USRP. To address this problem, synchronization should be guaranteed by adjusting the ultrasonic phase and monitoring the impact force; this task will be carried out in future work. In addition, future work will investigate the effects

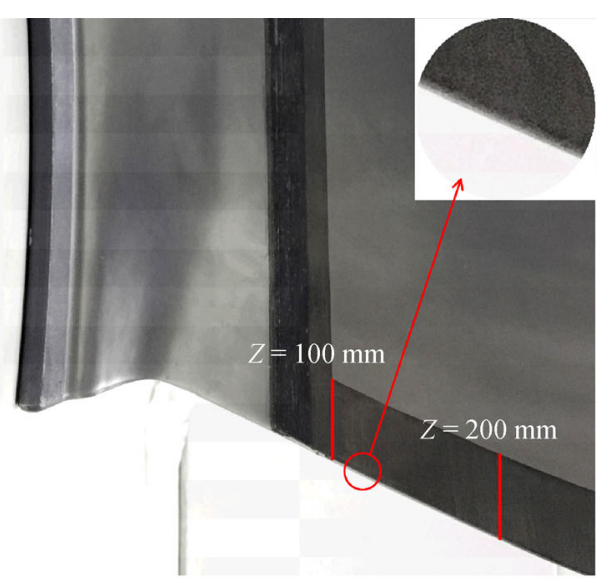

(b)

Fig. 31 (a) Strengthened region using the blade model to generate the machining path; (b) strengthened region using the proposed path generation method. 
of TS-USRP on the microstructure and surface integrity of aeroengine blades and the technological parameters of blade strengthening.

Acknowledgements The authors gratefully acknowledge the financial support extended by the National Natural Science Foundation of China (Grant Nos. 51975214, 51725503, and 51575183) and the 111 Project. Zhang $\mathrm{X} \mathrm{C}$ is also grateful for the support by the Major Program of the National Natural Science Foundation of Shanghai (Grant No. 2019-01-07-00-02E00068).

Open Access This article is licensed under Creative Commons Attribution 4.0 International License, which permits use, sharing, adaptation, distribution, and reproduction in any medium or format, as long as appropriate credit is given to the original author(s) and the source, a link is provided to the Creative Commons license, and any changes made are indicated.

Images or other third-party materials in this article are included in the article's Creative Commons license, unless indicated otherwise in a credit line to the material. If material is not included in the article's Creative Commons license and your intended use is not permitted by statutory regulation or exceeds the permitted use, you will need to obtain permission directly from the copyright holder.

To view a copy of this license, visit http://creativecommons.org/licenses/ by/4.0\%.

\section{References}

1. Yilmaz O, Gindy N, Gao J. A repair and overhaul methodology for aeroengine components. Robotics and Computer-Integrated Manufacturing, 2010, 26(2): 190-201

2. Qiao H C. Experimental investigation of laser peening on Ti17 titanium alloy for rotor blade applications. Applied Surface Science, 2015, 351: 524-530

3. Li P, Huang S, Xu H, et al. Numerical simulation and experiments of titanium alloy engine blades based on laser shock processing. Aerospace Science and Technology, 2015, 40(7): 164-170

4. Yao J, Zhang Q, Kong F, et al. Laser hardening techniques on steam turbine blade and application. Physics Procedia, 2010, 5: 399-406

5. Altenberger I, Nalla R K, Sano Y, et al. On the effect of deep-rolling and laser-peening on the stress-controlled low- and high-cycle fatigue behavior of $\mathrm{Ti}-6 \mathrm{Al}-4 \mathrm{~V}$ at elevated temperatures up to $550{ }^{\circ} \mathrm{C}$. International Journal of Fatigue, 2012, 44: 292-302

6. Xu G, Luo K Y, Dai F Z, et al. Effects of scanning path and overlapping rate on residual stress of 316L stainless steel blade subjected to massive laser shock peening treatment with square spots. Applied Surface Science, 2019, 481: 1053-1063

7. Zhang Y K, Lu J Z, Ren X D, et al. Effect of laser shock processing on the mechanical properties and fatigue lives of the turbojet engine blades manufactured by LY2 aluminum alloy. Materials \& Design, 2009, 30(5): 1697-1703

8. Hennig W, Feldmann G, Haubold T. Shot peening method for aerofoil treatment of blisk assemblies. Procedia CIRP, 2014, 13: 355-358

9. Benedetti M, Fontanari V, Winiarski B, et al. Residual stresses reconstruction in shot peened specimens containing sharp and blunt notches by experimental measurements and finite element analysis. International Journal of Fatigue, 2016, 87: 102-111
10. Montross C S, Wei T, Ye L, et al. Laser shock processing and its effects on microstructure and properties of metal alloys: A review. International Journal of Fatigue, 2002, 24(10): 1021-1036

11. Guo W, Sun R, Song B, et al. Laser shock peening of laser additive manufactured Ti6A14V titanium alloy. Surface and Coatings Technology, 2018, 349: 503-510

12. Fang Y, Li Y, He W, et al. Numerical simulation of residual stresses fields of DD6 blade during laser shock processing. Materials \& Design, 2013, 43: 170-176

13. Kalentics N, Boillat E, Peyre P, et al. 3D Laser Shock Peening-A new method for the 3D control of residual stresses in Selective Laser Melting. Materials \& Design, 2017, 130: 350-356

14. Wang T, Wang D, Liu G, et al. Investigations on the nanocrystallization of $40 \mathrm{Cr}$ using ultrasonic surface rolling processing. Applied Surface Science, 2008, 255(5): 1824-1829

15. Liu Y, Zhao X, Wang D. Determination of the plastic properties of materials treated by ultrasonic surface rolling process through instrumented indentation. Materials Science and Engineering A, 2014, 600: 21-31

16. Cai Z, Zhang X, Tu S. Effects of ultrasonic surface rolling process on microstructure and surface integrity of Ti-6Al-4V alloy. Materials for Mechanical Engineering, 2018, 42(1): 7-10 (in Chinese)

17. Kattoura M, Mannava S R, Qian D, et al. Effect of ultrasonic nanocrystal surface modification on elevated temperature residual stress, microstructure, and fatigue behavior of ATI 718Plus alloy. International Journal of Fatigue, 2018, 110: 186-196

18. Song D, Xue F, Wu D, et al. Iso-parametric path-planning method of twin-tool milling for turbine blades. International Journal of Advanced Manufacturing Technology, 2018, 98(9-12): 3179-3189

19. Li D, Zhang L, Yang X, et al. Research on the double-sided grinding and polishing machine tool system. In: Proceedings of 2010 IEEE International Conference on Information and Automation. Anchorage: IEEE, 2010, 1968-1971

20. Zhang L, Yang H R, Zhang Z J, et al. Modal analysis for a new double-side blade grinding machine. In: Proceedings of Applied Mechanics and Materials. Switzerland: Trans Tech Publications, 2012, 159: 156-159

21. Kopp R, Schulz J. Flexible sheet forming technology by doublesided simultaneous shot peen forming. CIRP Annals, 2002, 51(1): 195-198

22. Sakhvadze G Z, Pugachev M S, Kikvidze O G. Two-sided laser shock processing. Russian Engineering Research, 2017, 37(1): 4045

23. Zhang Y, Chen Z, Ning T. Reverse modeling strategy of aero-engine blade based on design intent. International Journal of Advanced Manufacturing Technology, 2015, 81(9-12): 1781-1796

24. Sun B, Li B. Laser displacement sensor in the application of aeroengine blade measurement. IEEE Sensors Journal, 2016, 16(5): 1377-1384

25. Xiao G, Huang Y, Fei Y. On-machine contact measurement for the main-push propeller blade with belt grinding. International Journal of Advanced Manufacturing Technology, 2016, 87(5-8): 17131723

26. Huang N, Bi Q, Wang Y, et al. 5-Axis adaptive flank milling of flexible thin-walled parts based on the on-machine measurement. 
International Journal of Machine Tools and Manufacture, 2014, 84: $1-8$

27. Nishikawa S, Ohno K, Mori M, et al. Non-contact type on-machine measurement system for turbine blade. Procedia CIRP, 2014, 24: $1-6$

28. Li F, Hitchens C, Stoddart D. A performance evaluation method to compare the multi-view point cloud data registration based on ICP algorithm and reference marker. Journal of Modern Optics, 2018, 65(1): $30-37$

29. Lu L X, Sun J, Li L, et al. Study on surface characteristics of 7050T7451 aluminum alloy by ultrasonic surface rolling process.
International Journal of Advanced Manufacturing Technology, 2016, 87(9-12): 2533-2539

30. Cheng M, Zhang D, Chen H, et al. Surface nanocrystallization and its effect on fatigue performance of high-strength materials treated by ultrasonic rolling process. International Journal of Advanced Manufacturing Technology, 2016, 83(1-4): 123-131

31. Zheng Y, Li G, Xu X, et al. Rolling normal filtering for point clouds. Computer Aided Geometric Design, 2018, 62: 16-28

32. Wang J, Oliveira M M. Filling holes on locally smooth surfaces reconstructed from point clouds. Image and Vision Computing, 2007, 25(1): 103-113 